\title{
Towards the Cosmic Distance Scale through Nebular SNe Ia
}

\author{
By P. RUIZ-LAPUENTE AND A. V. FILIP P E N K O $^{2}$ \\ ${ }^{1}$ Harvard-Smithsonian Center for Astrophysics \\ 60 Garden St., Cambridge, MA 02138, USA \\ ${ }^{2}$ Department of Astronomy, and Center for Particle Astrophysics, \\ University of California, Berkeley, CA 94720, USA
}

We give an update of current research on the use of nebular spectra of SNe Ia as distance indicators. Results of the application of the method to a group of SNe Ia are reported. We describe the status of the research including theoretical and observational requirements of the method. Our results point toward a shorter distance scale than methods based on the "standard candle" hypothesis for Type Ia SNe.

\section{Introduction}

The use of SNe Ia as "standard candles" to determine the extragalactic distance scale has been recurrently debated. The correlation found by Pskovskii $(1977,1984)$ and by Branch (1981) between the postmaximum decline rate of the light curve and the magnitude at maximum cast doubts concerning this method. The validity of the correlation was questioned by Boisseau \& Wheeler (1991), who found that such an effect might reflect contamination from the light of the underlying galaxy. But new evidence on differences in the light curve decline rate (Phillips 1993; Suntzeff, this volume) opens again the question of the correlation of magnitude at maximum and slope of the light curve soon after maximum. The value of the absolute magnitude of SNe Ia as a class loses much of its meaning if the considerable spread in magnitudes found in recent work is confirmed.

Uncertainties in the absolute magnitudes of SNe Ia are amplified by extinction. The discrepant "observationally-inferred" values obtained for SN 1986G (Phillips et al. 1992a; Della Valle \& Panagia 1992; Phillips 1993) show that when reddening is high the usual prescriptions to obtain this quantity from the color curves and from the equivalent width of the Na I D interstellar line towards the supernova give different results. These discrepancies occur even if the supernova is not highly affected by extinction, as in the case of SN $1991 \mathrm{~T}$ (see, for instance, values given in Filippenko et al. 1992, Ruiz-Lapuente et al. 1992, and Phillips et al. 1992a). All the methods using supernovae as distance indicators (Schmidt et al. 1992; Kirshner, this volume; Höflich, this volume) require an accurate estimate of reddening. When spectral modeling is involved, as in the Expanding Photosphere Method for Type II supernovae (Schmidt et al. 1992), a certain degree of cancellation is found in the effects of extinction on the final distance (reddening lowering the level of the flux but making the supernova appear cooler).

Ruiz-Lapuente (1992) and Ruiz-Lapuente \& Lucy (1992, hereafter Paper I) investigated the use of the nebular stage of the spectral evolution of SNe Ia to obtain a hint on nucleosynthesis, $E(B-V)$, and distance to particular events. We found that the bulk of the information contained in the optical spectra should make it possible to discriminate between intrinsic variations of the ratio of the luminosities of the lines (due to temperature effects and ionization effects) from extrinsic effects such as extinction. In the analysis of SN $1986 \mathrm{G}$ we proved that the correlation coefficients of the involved 
physical parameters $\left({ }^{56} \mathrm{Ni}, E(B-V)\right.$, and distance) were low enough to obtain these quantities separately. This was possible because of the various [Fe II] and [Fe III] lines distributed along the spectra.

In this paper we present further results, and we discuss the possibilities, potential difficulties, and improvements of the method. It is important to link the overall results with the present status of SN Ia research in what concerns the various models and different determinations of the relevant physical quantities.

\section{Applicability of the Method}

Several observational conditions are desired when using the nebular spectra of Type Ia supernovae for distance determinations. (Hereafter we refer to this approach as the Nebular SN Ia Method.) First, there should be no differential losses in flux between the blue and the red parts of the spectra, since greater losses in the blue result in overestimates of the reddening. These are minimized by aligning the slit along the parallactic angle, and by observing at low airmasses. Second, inaccurate subtraction of the galaxy background should also be avoided, since it typically tends to redden the spectrum and therefore affects the estimate of $E(B-V)$. The method is expected to work best when the supernova is in a region where contamination from the galaxy is low. Third, comparison of the nebular spectra with observed light curves is required to set the absolute fluxes of the spectra. The narrow slit rarely includes all the light, and conditions can vary from night to night or even during the course of a single night. A check of the consistency of the colors obtained from the spectra with those derived from the light curves is also recommended.

\section{The Sample of SNe Ia in the Nebular Phase}

A number of spectra of SNe Ia are available in the nebular phase: SN 1987L in NGC 2336, SN $1991 \mathrm{~T}$ in NGC 4527, SN 1989M in NGC 4579, SN 1990N in NGC 4639, SN 1992A in Fornax A, and two SNe Ia in the Centaurus Group (SN 1972E in NGC 5253 and SN 1986G in NGC 5128) are currently the best observed ones. A SN Ia at redshift $z=0.011$, SN 1991aa in an anonymous galaxy also has adequate spectra for the use of the method. For other SNe Ia, data are available over limited wavelength coverage, or too soon after maximum brightness for the purposes of our study (which is limited to the phase where continuum radiation is negligible). The data now available to us come mainly from observations done at the MMT (CfA archive), Lick Observatory, CTIO, and the WHT (La Palma Observatory). There is work in progress to homogenize the sample of spectra by using light curves; this will allow us to obtain results of higher accuracy.

We review, now, the first steps towards the determination of the cosmic distance scale through SNe Ia in the nebular phase. They are limited to two SNe Ia in the Centaurus Group, a SN Ia in the Virgo Cluster, and a SN Ia beyond the Virgo Cluster. We present here the results obtained by applying our method to the data. These can be seen in Table 1, where comparisons are made with results obtained from other methods.

The application of the method to SN 1991T, for which spectra from different observatories are available, gives a distance towards its host galaxy, NGC 4527, between 12 and $14 \mathrm{Mpc}$. For these data, the flux level for the different nebular spectra refers to calibration done by spectrophotometry with standard stars. The lack of homogeneity in the calibration of the spectra enters in the quoted error.

These results confirm that SN $1991 \mathrm{~T}$ is on the near side of the Virgo Cluster, as compared with the distances obtained by applying the Nebular SN Ia Method to other 
TABLE 1. Distances to SNe and their host galaxies through the Nebular SN Ia Method

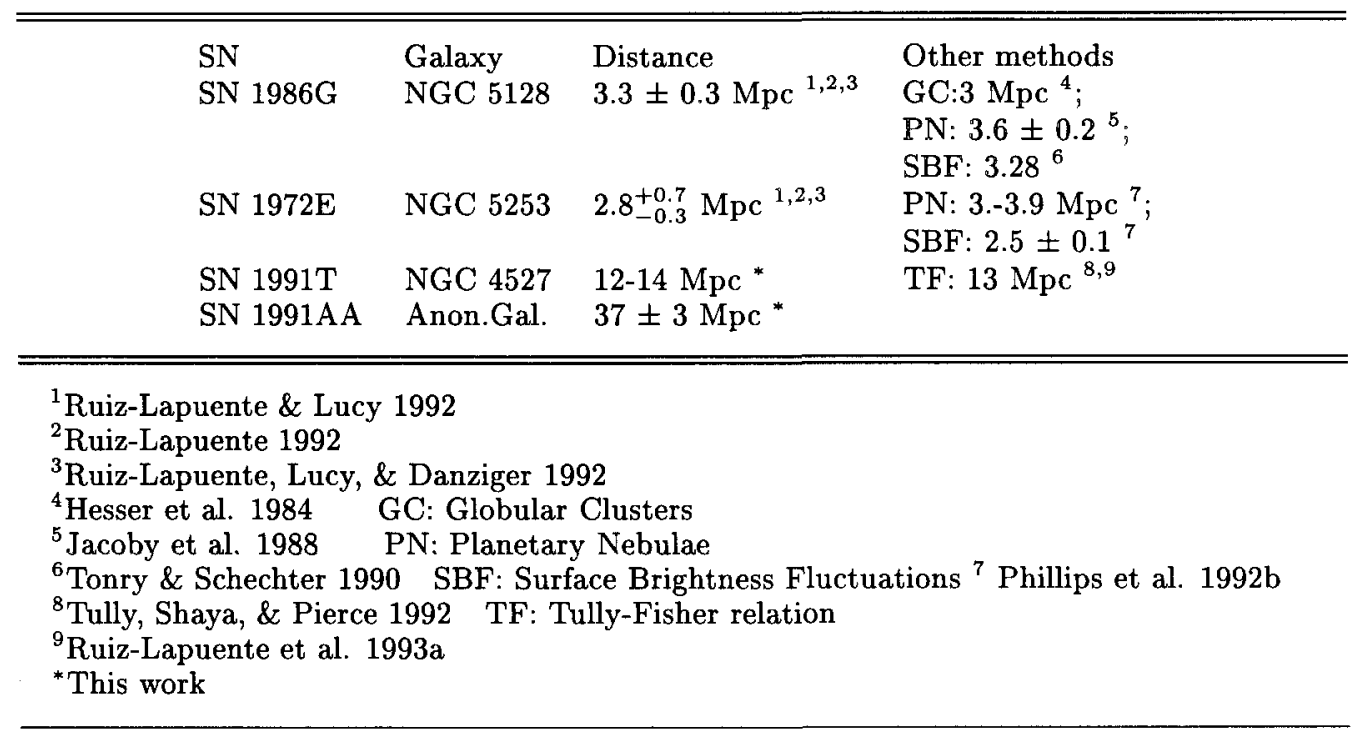

SNe Ia such as SN 1990N. The implied distance for NGC 4527 from the use of the nebular spectra of SN 1991T is consistent with the distance determination by Tully, Shaya, \& Pierce (1992) using the Tully-Fisher relation. It also agrees with the analysis of the diagram $m_{B}$-flux $(847 \mathrm{keV}$ ) for SN 1991T (Ruiz-Lapuente et al. 1993a). The results of the Nebular SN Ia Method to the most distant SN Ia observed around 250 days after explosion, SN 1991aa ( $\left.c z=3300 \mathrm{~km} \mathrm{~s}^{-1}\right)$, suggest that the host galaxy is at a distance of $37 \pm 3 \mathrm{Mpc}$. This galaxy is beyond the Virgo Cluster and does not seem to be associated with any known group. Thus, a tentative corrected Hubble flow velocity around 3100 $\pm 200 \mathrm{~km} \mathrm{~s}^{-1}$ would give $\mathrm{H}_{0}=84 \pm 10 \mathrm{~km} \mathrm{~s}^{-1}$. This value needs to be confirmed by applying the method to other SNe Ia and examining its uncertainties. In Figure 1 we show the calculated spectra compared with the observed spectra around 275 days after explosion for SN 1991T and around 250 days after explosion for SN 1991aa; analysis of the Fe-peak nucleosynthesis in both $\mathrm{SNe}$ was published by Ruiz-Lapuente \& Filippenko (1993).

Since distance and reddening are important in setting the absolute flux scale of the spectra, we include in Table 2 the reddening obtained for every SN Ia of this set. In Table 3 we present the amount of ${ }^{56} \mathrm{Ni}$ in the Fe-peak region (the former nuclear statistical equilibrium core) derived for every SN Ia. The errors quoted come from the statistical least-squares analysis. For SN 1972E, lack of direct access to the computer-readable data precluded the application of the least-squares analysis. Our inspection of the data as published in Meyerott (1980) gave a distance to NGC 5253 between 2.5 and $3.5 \mathrm{Mpc}$ and a low reddening, from 0 to 0.1 magnitudes (Paper I). A distance to NGC 5128 (SN $1986 \mathrm{G}$ ) between 3 and $3.6 \mathrm{Mpc}$ is obtained from the least-squares procedure, and the supernova seems to be highly reddened. 

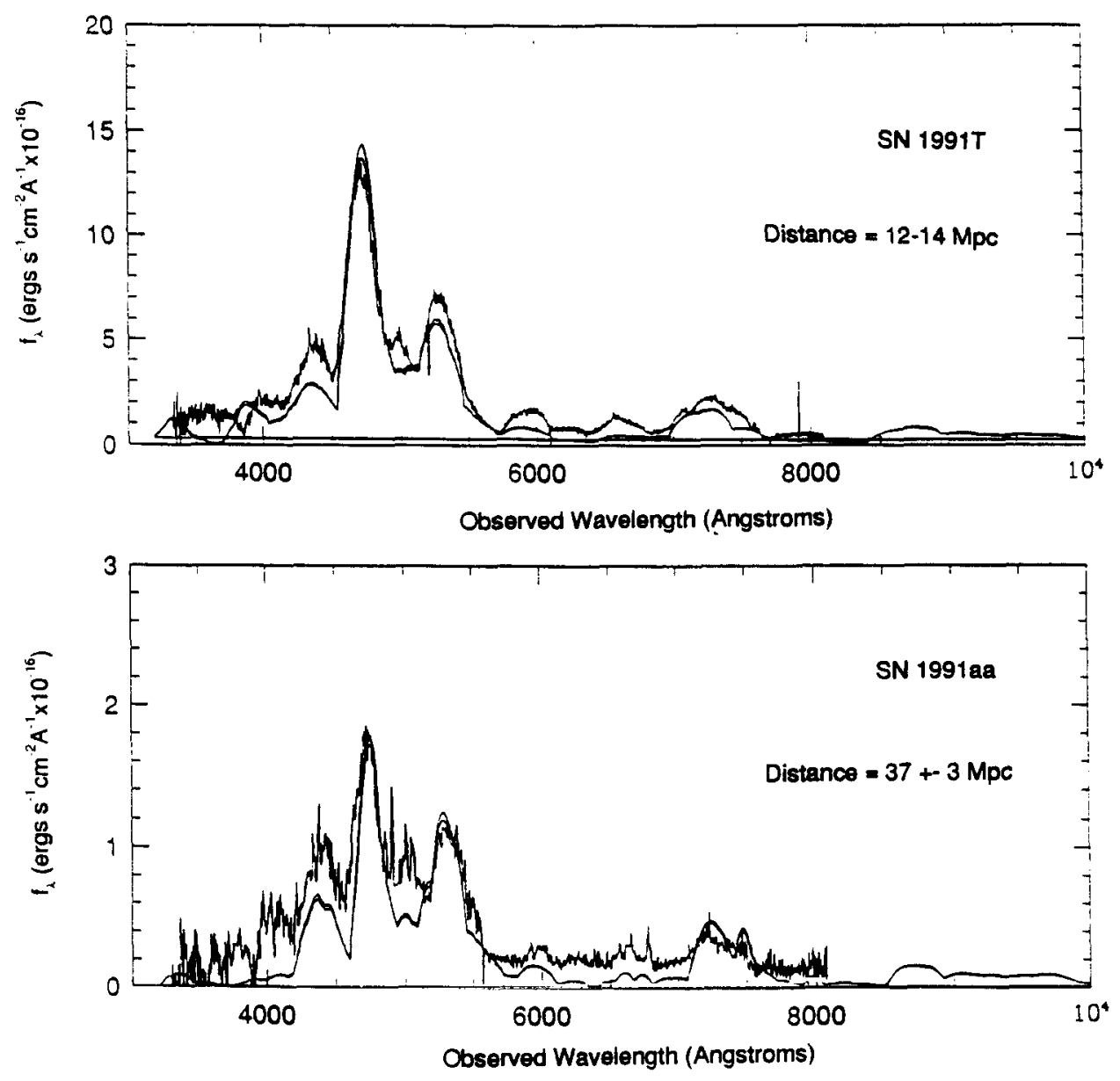

Figure 1. Observed and theoretical spectra for SN 1991T (top) and SN 1991aa (bottom) in the nebular phase (see text). The distance inferred by applying the method is $12-14 \mathrm{Mpc}$ for SN $1991 \mathrm{~T}$ in NGC 4527 and $37 \pm 3 \mathrm{Mpc}$ for SN 1991aa in an anonymous galaxy at an observed redshift of $z=0.011$.

\section{Theoretical Issues}

Internal errors could arise from our theoretical modeling. However, if we had failed to correctly describe the physical stage of the SN Ia ejecta, we would have been unable to reproduce the overall spectrum: wrong estimates of the electron temperature should manifest themselves in poor fits to the various emission lines in the different bands. Given that the ionization state has been fairly well determined from the spectra, we don't think our model can be grossly in error. To obtain the correct electron temperature from a model, we must know the efficiency in the deposition of the energy in gamma rays and positrons in the envelope. Theoretical modeling done so far seems to give agreement on the electron temperature for a given underlying model (Axelrod 1980; Meyerott 1978, 1980; comparison of our results with those from the code of Eastman \& Pinto 1993). The electron temperature is also well determined from the luminosity of the [Fe III] and [Fe II] lines in a model-independent basis, as done in the nebular analysis. The overall modeling suggests that the dominant ion of Fe in the nebular phase is Fe III. Significant amounts 
TABLE 2. Reddening $\mathrm{E}(\mathrm{B}-\mathrm{V})$

\begin{tabular}{llll}
\hline \hline SN & Galaxy & Reddening & Other methods \\
\hline SN 1986G & NGC 5128 & $1.09 \pm 0.02^{1,2,3}$ & $0.68-1.24^{10,11,12}$ \\
SN 1972E & NGC 5253 & $0.05 \pm 0.05^{1,2,3}$ & $0.02^{12}$ \\
SN 1991T & NGC 4527 & $0.1-0.2^{*}$ & $0.1-0.3^{9,13,14,15}$ \\
SN 1991AA & Anon.Gal. & $0.13 \pm 0.04^{*}$ & \\
& $\left(\mathrm{v}=3300 \mathrm{~km} \mathrm{~s}^{-1}\right)$ & & \\
\end{tabular}

${ }^{10}$ Di Serego Alighieri and Ponz 1987

${ }^{11}$ Phillips et al. 1987

${ }^{12}$ Della Valle and Panagia 1992

${ }^{13}$ Filippenko et al. 1992

${ }^{14}$ Ruiz-Lapuente et al. 1992a

${ }^{15}$ Phillips et al. 1992a

*This work

TABLE $3 \cdot{ }^{56} \mathrm{Ni}$ mass $^{16}$

\begin{tabular}{llll}
\hline \hline SN & Galaxy & ${ }^{56} \mathrm{Ni}$ mass \\
SN 1986G & NGC 5128 & $0.38 \pm 0.03 \mathrm{M}_{\odot}$ \\
SN 1972E & NGC 5253 & $0.5-0.6 \mathrm{M}_{\odot}$ \\
SN 1991T & NGC 4527 & $0.7-0.8 \mathrm{M}_{\odot}$ \\
SN 1991AA & Anon.Gal. & $0.6 \mathrm{M}_{\odot}$ \\
& $\left(\mathrm{v}=3300 \mathrm{~km} \mathrm{~s}^{-1}\right)$ & \\
\hline
\end{tabular}

${ }^{16}$ Ruiz-Lapuente and Filippenko 1993

of Fe II and Fe IV are also predicted from the ionization balance (taking into account photoionization coming from cascade emission towards the ground term; Paper I). On the other hand, Axelrod (1980) noticed that the lack of [Fe VI] and [Fe VII] lines in the observed optical spectra of SN 1972E (Kirshner \& Oke 1975) reflected the inadequacy of some models of SN Ia explosions to reproduce the ionization state of this supernova. The proportion of Fe II should be, according to our investigations, not lower than $\sim 10 \%$ of the total Fe abundance, in order to reproduce the optical spectra. Eastman reported at this colloquium preliminary results pointing towards a substantial reduction of Fe II in the calculated spectra when taking into account new photoionization cross-sections from excited levels. Such an increase of the ionization towards higher amounts of Fe III and Fe IV, and a much smaller fraction of Fe II, should be further investigated by different methods. At the time of the conference we had not yet evaluated the impact of the new photoionization cross-section in our determinations. However, according to our analyses, a failure to reproduce the observed data would arise if $\mathrm{Fe} \mathrm{II} \mathrm{is} \mathrm{drastically} \mathrm{reduced,} \mathrm{and}$ hardly any model (lowering the amount of ${ }^{56} \mathrm{Ni}$ ) would satisfy the different constraints. Fortunately, ionization can be well constrained on an empirical basis through spectra covering a wide wavelength range. Just recently, observations made with HST provide 
the ultraviolet part of the spectrum of SN 1992A (work in progress of the Supernova INtensive Studies [SINS] group); this will significantly constrain the proportions of all the ions.

Additional issues concern the population of the energy levels and the rate of collisional excitation over spontaneous radiative decays. We use in our calculation the most recent values available for collision strengths of [Fe III] and [Fe II] lines. The first calculations of the [Fe III] values were done by Garstang et al. (1978). More recent calculated collision strengths have been obtained by Berrington et al. (1991). From a comparison of previous and new values, one sees that significant discrepancies are found in the values of the weaker lines (i.e., transitions ${ }^{5} \mathrm{D}-{ }^{3} \mathrm{H}$ and ${ }^{3} \mathrm{H}-{ }^{3} \mathrm{P}$ ), which are not contributing significantly to the spectra). The values for the transitions ${ }^{5} \mathrm{D}-{ }^{3} \mathrm{~F}$ and ${ }^{5} \mathrm{D}-{ }^{3} \mathrm{P}$, which give rise to the $4600 \AA$ and the $5200 \AA$ emission seen in the spectra, are in much better agreement in both calculations (values by Berrington et al. 1991 and Garstang et al. 1978). The discrepancies between new and old values translate into a final $4 \%$ increase in distance (P.R.L. 1993, result presented at the Aspen Winter Conference on the Cosmic Distance Scale). Old distance estimates from this method towards SN 1986G and SN 1972E should change now (with the present values) to $4 \%$ larger distances. The collision strengths for [Fe II] by Pradhan \& Berrington (1993) seem to show some discrepancies as compared with previous values by Nussbaumer \& Storey (1980) and by Nussbaumer et al. (1981). A full analysis of the effect of the reported discrepancies for several transitions, as compared with previous work, is one of our future aims. The bulk of the work being developed by the Opacity Project on atomic data for Fe (Berrington et al. 1987) will help to improve the accuracy of our results.

\section{SN Ia Diversity and Distance Scale}

Current models of SN Ia are challenged by recent observations (see, for instance, Filippenko in this conference, Suntzeff in this conference, Ruiz-Lapuente et al. 1993b, Wheeler, Swartz, \& Harkness 1993). Our first approach has been to investigate the one-zone treatment, which reflects the fact that the Fe-peak region can be well modeled by describing the bulk of emission as arising from the zone where ${ }^{56} \mathrm{Ni}$ is the dominant component. We tested the hypothesis that calculations of the nebular spectra of SNe Ia are well reproduced by the theoretical spectrum of the region where ${ }^{56} \mathrm{Ni}$ is the dominant component of the ejecta for the case of SN 1991bg in NGC 4374. The two different approaches have given similar results in the amount of ${ }^{56} \mathrm{Ni}$ synthesized by the explosion (Ruiz-Lapuente et al. 1993b).

One advantage of this type of method is that it addresses the modeling of SNe Ia on an individual basis, thus showing in every case how well we are able to reproduce the spectra, and not being bound to any particular sample of hydrodynamical models. The set of SNe Ia analyzed thus far points to a shorter distance scale than other methods that use the "standard candle" hypothesis for SNe Ia. Differences in spectroscopic evolution, as well as correlations between the magnitude at maximum and the decline rate of the light curve, suggest that we view the "standard candle" hypothesis with caution. The approach presented in our earlier work (Paper I) has the advantage of being model independent and also not relying on the assumption of "normality" within the class of Type Ia SNe. 


\section{Acknowledgements}

P.R.L. acknowledges financial assistance through STScI grant GO-2563.01-87A. A.V.F. is supported by NSF grants AST-8957063 and AST-9115174, as well as by the Center for Particle Astrophysics at U.C. Berkeley (NSF Cooperative Agreement AST-8809616).

\section{REFERENCES}

Axelrod, T.S. 1980, Ph.D. Thesis, Univ. California at Santa Cruz.

Berrington, K.A., Burke, P.G., Butler, K., Seaton, M.J., Storey, P.J., Taylor, K.T., \& Yu Yan. 1987, J.Phys.B, 20, 6379.

Berrington, K.A., Zeippen, C.J., Le Dourneuf, M., Eissner, W., \& Burke, P.G. 1991, J.Phys.B, 24,3467 .

Boisseau, J.R., \& Wheeler, J.C. 1991, AJ, 101, 1281.

Branch, D. 1981, ApJ, 248, 1076.

Della Valle, M., \& Panagia, N. 1992, AJ, 104, 696.

di Serego Alighieri, S., \& Ponz, J. D. 1987, in ESO Workshop on the SN 1987A, ed. I. J. Danziger (Garching: ESO), p. 545.

Eastman, R.G. \& Pinto, P.A. 1993 ApJ, 412, 731.

Filippenko, A.V., et al. 1992, AJ, 104, 1543.

Garstang, R. H., Robb, W.D., \& Rountree, S.P. 1978, ApJ, 222, 384.

Hesser, J.E., Harris, H.C., van den Bergh, S., and Harris, G.L.H. 1984, ApJ, 276, 491.

Jacoby, G.H., Ciardullo, R., \& Ford, H.C. 1988, in The Extragalactic Distance Scale, Proceedings of the ASP 100th Anniversary Symposium, ASP Conf. Ser., Vol. 4., ed. S. van den Bergh and C.J. Pritchet (Provo, UT, Astron. Soc. Pacific), p. 42.

Kirshner, R.P., \& Oke, J.B. 1975, ApJ, 200, 574.

Meyerott, R.E. 1978, ApJ, 221, 975.

Meyerott, R.E. 1980, ApJ, 259, 257.

Nussbaumer, H., \& Storey, P.J. 1980, A\&A, 89, 308.

Nussbaumer, H., Pettini, M., \& Storey, P.J. 1981, A\&A, 102, 351.

Phillips, M.M. 1993, ApJ, 413, L105.

Phillips, M.M., et al. 1987, PASP, 99, 592.

Phillips, M.M., Wells, L.A., Suntzeff, N.B., Hamuy, M., Leibundgut, B., Kirshner, R.P., \& Foltz, C.B. 1992a, AJ, 103, 1632.

Phillips, M.M., Jacoby, G.H., Walker, A.R., Tonry, J.L., \& Ciardullo, R. 1992b, BAAS, 24, 749.

Pradhan, A.K., \& Berrington, K.A. 1993, J.Phys.B, 26, 157.

Pskovskii, Y.P. 1977, Sov. Astron., 21, 675.

Pskovskii, Y.P. 1984, Sov. Astron., 28, 658.

Ruiz-Lapuente, P. 1992, PhD Thesis, University of Barcelona.

Ruiz-Lapuente, P., Cappellaro, E., Turatto, M., Gouiffes, C., Danziger, I.J., Della Valle, M., \& Lucy, L.B. 1992a, ApJ, 387, L33.

Ruiz-Lapuente, P., Lucy, L.B., \& Danziger, I.J. 1992b, Mem. S.A.It., 63, 233,

Ruiz-Lapuente, P., \& Filippenko, A.V. 1993, in Origin and Evolution of the Elements, ed. N. Prantzos, E. Vangioni-Flam, \& M. Cassé (Cambridge: Cambridge Univ. Press), p. 318.

Ruiz-Lapuente, P., Lichti, G.G., Lehoucq, R., Canal, R., \& Cassé. M. 1993a, ApJ, 417, 547

Ruiz-Lapuente, P., Jeffery, D.J., Challis, P.M., Filippenko, A.V., Kirshner, R.P., Ho, L.C., Schmidt, B.P., Sánchez, F., \& Canal, R. 1993b, Nature, 365, 28

Ruiz-Lapuente, P., \& Lucy, L.B. 1992, ApJ, 400, 127 (Paper I). 
40 P. Ruiz-Lapuente \& A. V. Filippenko: Cosmic Distances \& Nebular SNe Ia

Schmidt, B.P., Kirshner, R.P., \& Eastman, R.G. 1992, ApJ, 395, 366.

Tonry, J.L., \& Schechter, P.L. 1990, AJ, 100, 1794.

Tully, R.B., Shaya, E.J., \& Pierce, M.J. 1992, ApJS, 80, 479.

Wheeler, J.C., Swartz, D.A., \& Harkness, R.P. 1993, Phys. Rep., 227, 113. 